\title{
A Novel Z-Type Self-Balancing Modular Multilevel Converter for Flywheel Driving Applications
}

\author{
Shuoqi Cheng ${ }^{1}$, Tong Zheng ${ }^{1}$, Congzhe Gao ${ }^{1, \mathrm{a}}$, Jingliang $\mathrm{Lv}^{2}$ and Xinjian Jiang ${ }^{2}$ \\ ${ }^{1}$ School of Automation, Beijing Institute of Technology, Beijing, 100081, China \\ ${ }^{2}$ Department of Electrical Engineering, Tsinghua University, Beijing, 100084, China.
}

\begin{abstract}
With the development of flywheel technology, the speed and the voltage level of flywheel continue to increase. The Z-type self-balancing modular multilevel converter can have a wide frequency range at medium voltage and is suitable for flywheel driving application. However, the voltage and current characteristics of the flywheel and the variable frequency drive still affect capacitor voltage balance in each phase-leg. The new balance of capacitor voltage can be achieved by controlling the circulating current. This paper proposed a control algorithm of the novel Z-type self-balancing modular multilevel converter for flywheel driving applications. Simulation results are presented to validate the theoretical analysis.
\end{abstract}

\section{Introduction}

Distributed energy system is an advanced model for future energy development. The distributed energy system is referring to the combination with various integrated or stand-alone modular power generation devices with a capacity of several $\mathrm{kW}$ to $50 \mathrm{MW}$ and energy storage systems. Flywheel energy storage system is a new type of energy storage system which stores electrical energy as kinetic energy of the rotating flywheel and discharges the energy by converting kinetic energy into electrical energy[1,2]. As the capacity of the flywheel increases, the voltage level also increases. At present, most researches on FESS focus on structure of flywheel[3,4]. There are relatively few studies on the application of the medium voltage modular multilevel converter to the flywheel.

Common multilevel topologies are divided into four basic topologies: the neutral point clamped (NPC)[5], the flying capacitor (FC)[6], the cascade H-bridge (CHB)[7], and the modular multilevel converter (MMC) $[8,9]$. The number of diodes in NPC and capacitors in FC increase drastically with the number of levels. CHB needs many separated DC sources which limits its application. Less number of power switches or capacitors, and only one DC source are used in MMC. Because the capacitors of submodules are floating to generate multilevel output, the capacitor voltage balancing issue is the major problem to the MMC.

Reference [10] proposes a novel Z-type modular multilevel converter(ZTMMC) with capacitor voltage self-balancing capability. With the help of clamping circuits in the Z-type submodules (ZT-SMs), inner energy loops are created to balance the capacitor voltages. Due to simple control algorithm and perfect voltage balancing performance, the ZTMMC is a suitable solution for flywheel energy storage system.

This paper proposes a control algorithm of the novel Z-type modular multilevel converter for flywheel driving applications. The circulating current can influence the capacitor voltage in each phase-leg. The capacitor voltage balance in each phase-leg is achieved by controlling the circulating current. For flywheel drive, the circulation current fundamental angle need compensation.

The rest of the paper is organized as follows. Section 2 introduces topology configuration for flywheel energy storage system. Capacitor voltage in each phase-leg is analysed and its control algorithm for flywheel is proposed in Section 3. Then, Section 4 introduces simulation results based on MATLAB. A conclusion is presented in Section 5.

\section{Topology Configuration}

A topology for flywheel driving applications with ZType submodules is shown in Figure 1. It includes threephase unit, each phase contains two upper and lower bridge arms and two bridge arm inductances. One side of the upper and lower bridge arms passes through the bridge arm inductance connected as the AC output side, and the other side of the upper and lower bridge arms are connected in parallel to the common DC bus. Each bridge arm is connected by 5 cascaded Z-Type submodules.

$\overline{{ }^{a} \text { Corresponding author: gcz_fly@163.com }}$ 


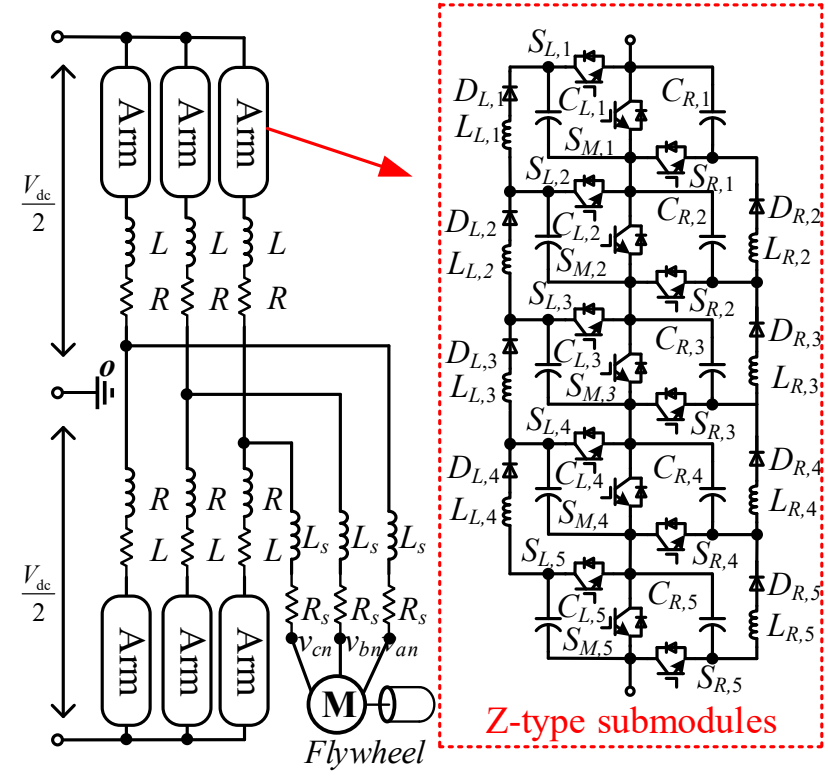

Figure 1. Topology of a three-phase MMC for flywheel based on the ZT-SM.

The bridge arm voltage of the Z-type self-balancing modular multilevel converter is synthesized from the output voltages of 5 submodules. For a single submodule, the power level of the left and right clamping circuits of the Z-type sub-module is much lower than that of the main power circuit. Therefore, only the main power circuit of the Z-type submodule is analysed, and its switching mode is shown in Figure 2. In the figure, $v_{C p x, j}(p=a, b, c ; x=u, l ; 1 \leqslant j \leqslant 5)$ is the capacitor voltage of the submodules in the bridge arm, the left and right capacitors in the submodules are equal and half of the total capacitance of the submodules, which is shown in formula (1).

$$
C_{L}=C_{R}=\frac{C}{2}
$$

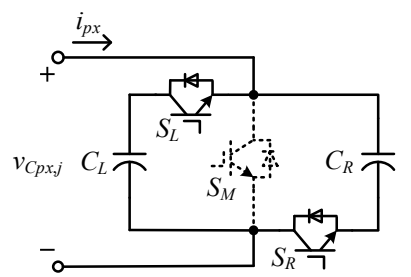

(a) on circuit, $S_{p x, j}(t)=1$

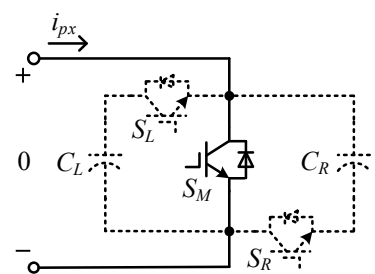

(b) Bypass, $S_{p x, j}(t)=0$
Figure 2. Submodule switching mode.

The switch function of the submodule can be expressed as:

$S_{p x, j}= \begin{cases}1, & S_{R} \text { and } S_{L} \text { are on, } S_{M} \text { is off. } \\ 0, & S_{M} \text { is on, } S_{R} \text { and } S_{L} \text { are off. }\end{cases}$

The output voltage of submodule can be expressed as:

$$
v_{p x, j}=S_{p x, j} v_{C p x, j}
$$

When the submodule is on circuit, the capacitor voltage of the submodule follows the change of the bridge arm current, which can be expressed as

$$
C \frac{d v_{C p x, j}}{d t}=S_{p x, j} i_{p x}
$$

Each bridge arm of the Z-type self-balancing modular multilevel converter is composed of 5 Z-type submodules in series. According to formula (3), the output voltage of the bridge arm can be expressed as

$$
v_{p x, j}=\sum_{j=1}^{5} v_{p x, j}=\sum_{j=1}^{5}\left(S_{p x, j} v_{C p x, j}\right)
$$

The cascaded bridge arm of the Z-type submodule has the characteristic of internal capacitors selfbalancing, ignoring the ripple of the switching frequency, and the capacitor voltage can be expressed as

$$
v_{C p x, 1}=v_{C p x, 2}=v_{C p x, 3}=v_{C p x, 4}=v_{C p x, 5}=v_{C p x}
$$

Define the number of sub-module inputs in the bridge arm as $n_{p x}$.

$$
n_{p x}=\sum_{j=1}^{5} S_{p x, j}
$$

Formula (4) and (5) can be simplified as

$$
\left\{\begin{array}{c}
v_{p x}=n_{p x} v_{C p x} \\
N C \frac{d v_{C p x}}{d t}=n_{p x} i_{p x}
\end{array}\right.
$$

The output voltage of the bridge arm can be equivalent to a controlled voltage source, and the current flowing through the capacitor can be equivalent to a controlled current source. The single-phase equivalent circuit of the Z-type self-balancing modular multilevel converter is shown in the figure 3 .

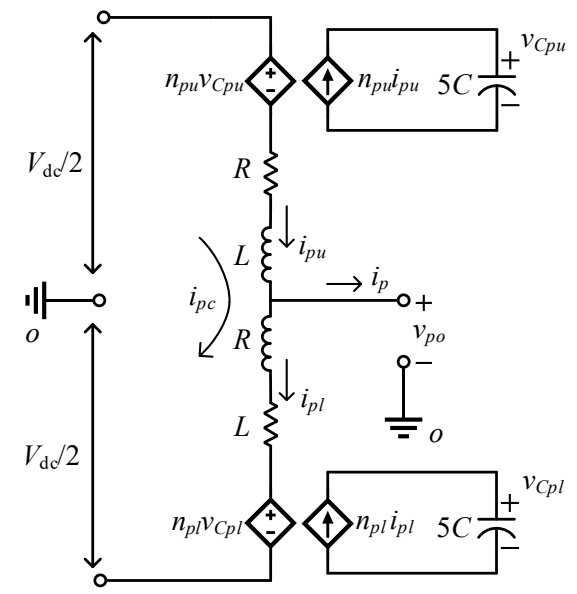

Figure 3. The single-phase equivalent circuit of the ZTMMC.

According to Kirchhoff's voltage law, formula (9) can express voltage loop of upper and lower bridge arms. 


$$
\left\{\begin{array}{l}
n_{p u} v_{C p u}+R i_{p u}+L \frac{d i_{p u}}{d t}=\frac{V_{d c}}{2}-v_{p o} \\
n_{p l} v_{C p l}+R i_{p l}+L \frac{d i_{p l}}{d t}=\frac{V_{d c}}{2}+v_{p o}
\end{array}\right.
$$

In the figure $3, i_{p c}$ is the internal circulation current of the phase. According to Kirchhoff's current law, formula (10) can be derived.

$$
\left\{\begin{array}{c}
i_{p c i r}=\frac{i_{p u}+i_{p l}}{2} \\
i_{p}=i_{p u}-i_{p l}
\end{array}\right.
$$

The mathematical model of the Z-type self-balancing modular multilevel converter can be expressed as

$$
\frac{d}{d t}\left[\begin{array}{c}
v_{C p u} \\
v_{C p l} \\
i_{p c} \\
i_{p}
\end{array}\right]=\left[\begin{array}{cccc}
0 & 0 & \frac{n_{p u}}{N C} & \frac{n_{p u}}{2 N C} \\
0 & 0 & \frac{n_{p l}}{N C} & -\frac{n_{p l}}{2 N C} \\
-\frac{n_{p u}}{2 L} & -\frac{n_{p l}}{2 L} & -\frac{R}{L} & 0 \\
-\frac{n_{p u}}{L} & -\frac{n_{p l}}{L} & 0 & -\frac{R}{L}
\end{array}\right]\left[\begin{array}{c}
v_{C p u} \\
v_{C p l} \\
i_{p c} \\
i_{p}
\end{array}\right]+\left[\begin{array}{c}
0 \\
0 \\
\frac{V_{d c}}{2 L} \\
2 v_{p o} \\
-\frac{1}{L}
\end{array}\right]
$$

\section{Control Algorithm for Flywheel}

\subsection{Capacitor Voltage in Each Phase-Leg}

In closed-loop modulation, the number of input submodules in the upper and lower bridge arms is obtained by dividing the reference voltage of the bridge arm output by the actual value of the sub-module capacitor voltage.

$$
\left\{\begin{array}{l}
n_{p u}=\frac{v_{p u}^{r e f}}{v_{C p u}} \\
n_{p l}=\frac{v_{p l}^{r e f}}{v_{C p l}}
\end{array}\right.
$$

Substituting formula (12) into formula (11), the mathematical model of the internal bridge capacitor voltage of the ZTMMC under closed-loop modulation can be simplified as

$$
\left\{\begin{array}{l}
\frac{1}{2} N C \frac{d v_{C p u}^{2}}{d t}=v_{p u}^{r e f} i_{p u} \\
\frac{1}{2} N C \frac{d v_{C p l}^{2}}{d t}=v_{p l}^{r e f} i_{p l}
\end{array}\right.
$$

In order to simplify the analysis, the energy of the upper and lower arms of the ZTMMC is defined as

$$
\left\{\begin{array}{l}
E_{p u}=\frac{1}{2} N C v_{C p u}^{2} \\
E_{p l}=\frac{1}{2} N C v_{C p l}^{2}
\end{array}\right.
$$

According to formula (14), the total energy of the bridge arm and the differential energy of the bridge arm in each phase of the ZTMMC can be expressed as

$$
\left\{\begin{array}{l}
E_{p}^{\Sigma}=E_{p u}+E_{p l} \\
E_{p}^{\Delta}=E_{p l}-E_{p u}
\end{array}\right.
$$

Combining (13), (14), (15), the differential equations of the total energy of the bridge arm and the differential energy of the bridge arm in each phase of ZTMMC can be obtained as

$$
\left\{\begin{array}{l}
\frac{d E_{p}^{\Sigma}}{d t}=v_{p c}^{r e f} i_{p c}-v_{p o}^{r e f} i_{p} \\
\frac{d E_{p}^{\Delta}}{d t}=2 v_{p o}^{r e f} i_{p c}-v_{p c}^{r e f} i_{p}
\end{array}\right.
$$

It can be seen from formula (16) that the total energy of the bridge arm and the differential energy of the bridge arm in each phase of ZTMMC are simultaneously affected by the AC side current and the internal circulating current.

\subsection{Capacitor Voltage in Each Phase-Leg}

Since the AC side current of the ZTMMC is limited by the requirements of the application, the control of the capacitor voltage of the bridge arm is usually achieved by controlling its internal circulating current. The internal circulating current mathematical model of the Ztype self-balancing modular multilevel converter can be obtained as

$$
2 L \frac{d i_{p c i r}}{d t}+2 R i_{p c i r}=V_{d c}-v_{p c i r}^{r e f}
$$

Therefore, the internal circulating current control can be achieved by adjusting the sum of the output voltage of the upper and lower bridge arms. At the same time, the system transfer function of the internal circulating current of the Z-type self-balancing modular multilevel converter is a first-order system, which can be realized by PI control. The control block diagram is shown in Figure 4. $G_{c}(s)$ is the transfer function of PI control, and $G_{d}(s)$ represents the system delay caused by the PWM modulation, measurement and control cycle.

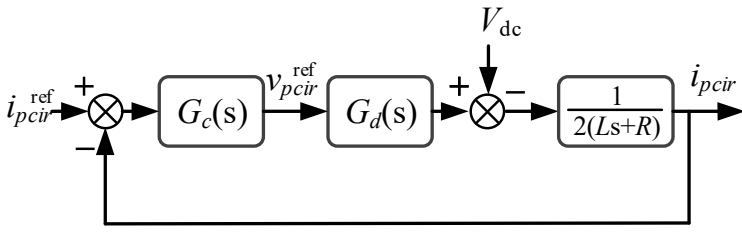

Figure 4. The circulating current control of the ZTMMC.

The reference value of the circulating current is controlled by the bridge arm energy outer loop. The overall control diagram of the converter is shown in Figure 5. The reference value of the circulating current can be divided into two parts, one part is generated by 
the total energy controller of the bridge arm, and it is to designed to maintain the stability of the total energy of the bridge arm; the other part is generated by the bridge arm differential energy controller, which is designed to suppress the deviation between the energy of the upper and lower bridge arms[12-14].

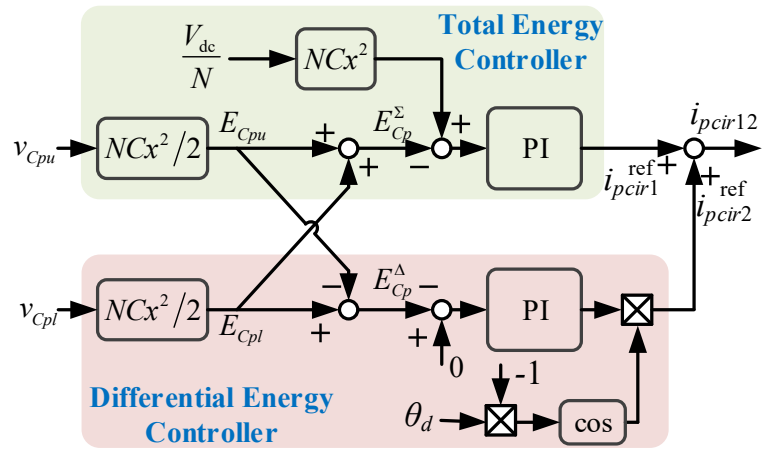

Figure 5. Circulating Control diagram of ZTMMC under closed-loop modulation

In the Figure $5, \theta_{d}$ is the magnetic field orientation angle of the flywheel. The capacitor voltage balance of the upper and lower bridge arms can be controlled by the internal circulating current, and the differential controller follows $\theta_{d}$, which can be realized in a wide output frequency. It is suitable for flywheel with variable output frequency.

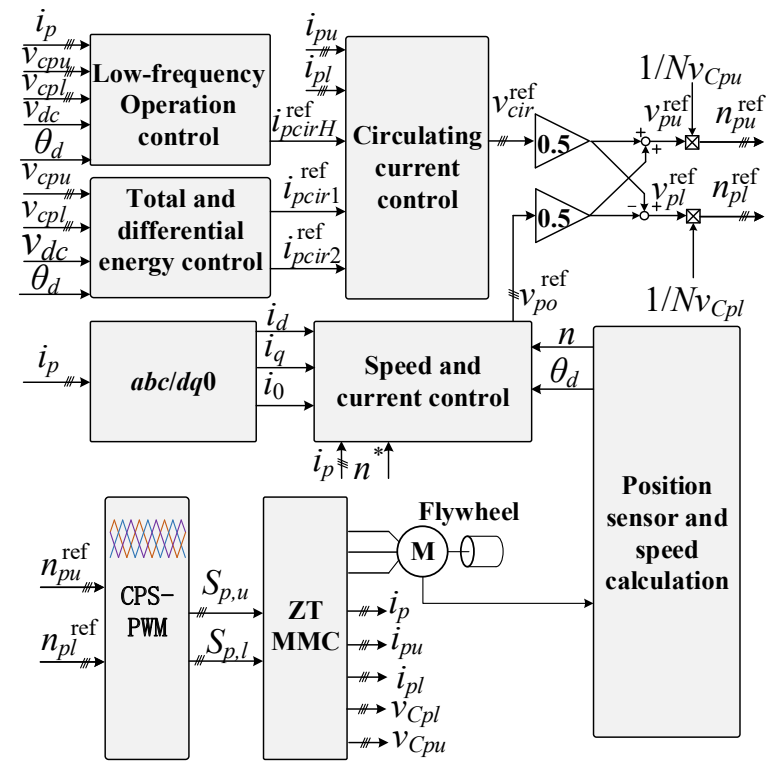

Figure 6. Control block diagram of the ZTMMC for flywheel driving application.

The control block diagram of the ZTMMC flywheel driving is shown in Figure 6. The phase current of the flywheel is transformed into $d q$ axis through coordinate transformation, and two PI controllers regulate the d-axis and $q$-axis current respectively. The reference of the $d$ axis current is set to be zero, while the reference of the $q$-axis current is given by the PI controller of the speed loop. Because the initial frequency in the start process of the flywheel is very low, the fundamental frequency circulating current injected by the differential energy controller is not effective. Under low frequency operation, high frequency circulating current injection is used[15].

\section{Simulation results}

In order to verify the voltage balance capability between the upper and lower bridge arms of the Z-type modular multilevel converter for flywheel driving application, this section builds a $3 \mathrm{kV}$ three-phase Z-type selfbalancing modular multilevel converter simulation model the Matlab/Simulink environment. The main simulation parameters are shown in Table 1.

Table 1. System Parameters in the Simulation

\begin{tabular}{ccc}
\hline \hline Symbol & Parameters & Values \\
\hline$V_{d c}$ & DC-link voltage & $3 \mathrm{kV}$ \\
$N$ & Total number of submodules per arm & 5 \\
$C_{\mathrm{SM}}$ & Submodule capacitance & $1.8 \mathrm{mF}$ \\
$L$ & Arm inductance & $2 \mathrm{mH}$ \\
$L_{i}$ & Clamping inductance & $47 \mu \mathrm{H}$ \\
$f_{c}$ & Carrier frequency & $5 \mathrm{kHz}$ \\
$f_{o}$ & Output frequency & $0 \sim 300 \mathrm{~Hz}$ \\
$U_{f}$ & RMS value of flywheel line voltage & $1.2 \mathrm{kV}$ \\
$P_{r}$ & Rated power & $500 \mathrm{~kW}$ \\
$p$ & Number of pole pairs & 2 \\
$n_{r}$ & Rated speed & $9000 \mathrm{rpm}$ \\
\hline \hline
\end{tabular}

\subsection{Formatting the title, authors and affiliations}

In order to verify the upper and lower arm capacitor voltage balance of the Z-type self-balancing modular multilevel converter, the capacitor voltage balance effect is shown in Figure 7.

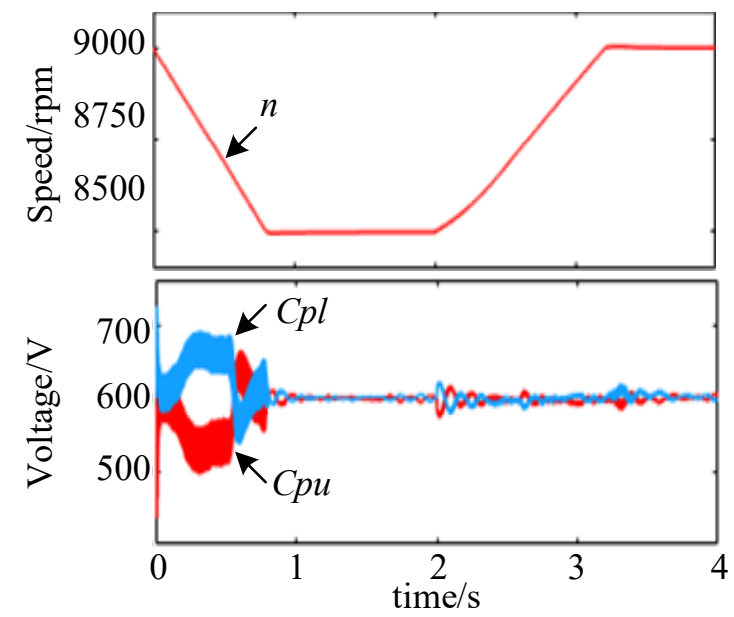

Figure 7. Speed and capacitor voltage waveform in simulation.

When $\mathrm{t}=0 \mathrm{~s}$, the speed of the flywheel is $9000 \mathrm{rpm}$, the reference speed is set to 8500rpm, and the flywheel discharges. When $\mathrm{t}=2 \mathrm{~s}$, the speed of the flywheel is $8500 \mathrm{rpm}$, the reference speed is set to $9000 \mathrm{rpm}$, and the flywheel stores energy.

Before the simulation time $t=0.5 \mathrm{~s}$, only the total energy of the arm capacitor voltage is enabled, after $t=0.5 \mathrm{~s}$, the differential energy control is enabled. Due to the self-balancing characteristics of the topology, the capacitor voltage of the upper and lower bridge arms reaches a stable value before the differential control is 
enabled, but there is still a small amount of deviation. This part of the deviation quickly converges by the differential energy controller.

when the speed reaches $8500 \mathrm{rpm}$ or $9000 \mathrm{rpm}$, the active component of the system is only the friction loss of the flywheel, and the capacitor voltage of the upper and lower bridge arms can still be stabilized at $600 \mathrm{~V}$.

\subsection{Steady State Operation Performance}

In order to verify steady state operation performance of the Z-type self-balancing modular multilevel converter, the capacitor voltage balance effect is shown in Figure 8.

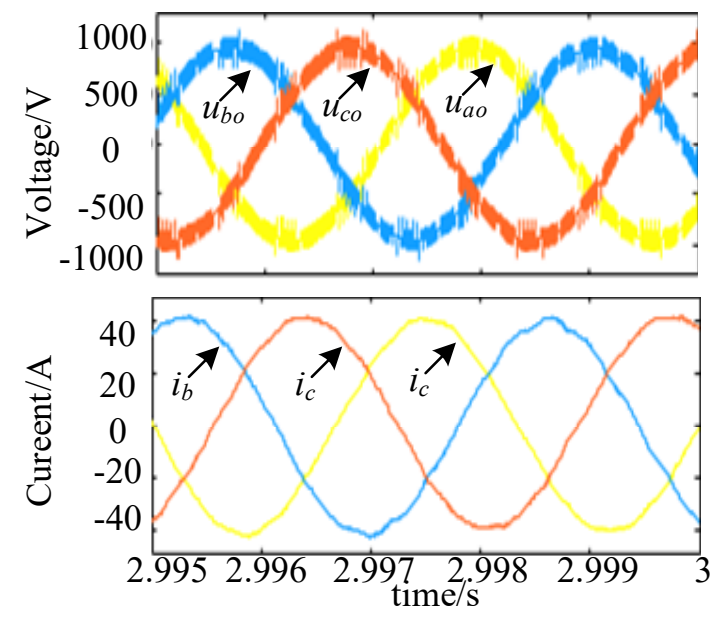

Figure 8. Three-phase output voltage and current waveform in simulation.

As shown in Figure 8, the voltage and current have a phase difference of approximately $90^{\circ}$, and the converter mainly outputs reactive power. The upper and lower bridge arm capacitor voltages still maintain $600 \mathrm{~V}$. It validates that the bridge arm capacitor voltage can still be well balanced when the Z-type self-balancing modular multilevel converter is working at a reactive state.

\section{Conclusion}

A capacitor voltage control algorithm of the novel Ztype self-balancing modular multilevel converter for flywheel driving applications is proposed in this paper. Each arm only needs to measure one capacitor voltage, and the balance of all capacitor voltages in all arms can be achieved under wide frequency through circulating current control. When the speed of the flywheel is stable and the converter outputs reactive power, the capacitor voltage can still be well balanced. With modular structure, simple control algorithm, four-quadrant operation, balancing performance, the Z-type MMC is a suitable solution for medium voltage flywheel driving applications.

\section{Acknowledgements}

This work is supported by the National Key Research and Development Program of China (Grant No. 2018YFB0905500)

\section{References}

1. Yeniu Q, Zonghui Y, Peng L, et al. Flywheel energy storage UPS power supply vehicle and its application in Beijing security of power supply. 2016 IEEE PES Asia-Pacific Power and Energy Engineering Conference (APPEEC): 2514-2522, (2016).

2. Mukoyama S, Nakao K, Sakamoto $H$, et al. Development of Superconducting Magnetic Bearing for $300 \mathrm{~kW}$ Flywheel Energy Storage System. IEEE Transactions on Applied Superconductivity, 27(4): 1-4, (2017).

3. Wei K, Dai X, Liu P. Model and balance of flywheel energy storage system with composite flywheel and rolling bearings. 2017 IEEE Conference on Energy Internet and Energy System Integration (EI2): 1-6, (2017).

4. Fang S, Lv Z, Chao G. Energy Density Improvement for Superconducting Flywheel Using Structure Optimization. 2020 IEEE International Conference on Applied Superconductivity and Electromagnetic Devices (ASEMD): 1-2,(2020).

5. Nabae A, Takahashi I, Akagi H. A New NeutralPoint-Clamped PWM Inverter. IEEE Transactions on Industry Applications, IA-17(5): 518-523, (2008).

6. Meynard T A. Multi-level conversion: high voltage choppers and voltage-source inverters. Proc.ieee Power Electronics Specialist Conf, (1992).

7. Peng, Fang, Zheng, et al. A multilevel voltagesource inverter with separate DC sources for static var generation. IEEE Transactions on Industry Applications, (1996).

8. Lesnicar A, Marquardt R. A new modular voltage source inverter topology. (2003).

9. R. Marquardt, "Stromrichterschaltungen mit verteilten Energiespeichern, "German Patent de10103031/24.01.2001,(Jan.24,2001).

10. Zheng T, Gao C, Liu X, et al. A Novel Z-Type Modular Multilevel Converter With Capacitor Voltage Self-Balancing for Grid-Tied Applications. IEEE Transactions on Power Electronics, 36(2): 1399-1411, (2021).

11. Thitichaiworakorn N, Hagiwara M, Akagi $H$. Experimental verification of a modular multilevel cascade inverter based on double-star bridge cells. IEEE Transactions on Industry Applications, 50 (1): 509-519,(2013).

12. Moon J-W, Kim C-S, Park J-W, Kang D-W, Kim JM. Circulating current control in MMC underthe unbalanced voltage. IEEE Transactions on Power delivery, 28 (3): 1952-1959, (2013). 
13. Li S, Wang X, Yao Z, Li T, Peng Z. Circulating current suppressing strategy for MMC-HVDC based on nonideal proportional resonant controllers under unbalanced grid conditions. IEEE Transactions on Power Electronics, 30 (1): 387-397, (2014).

14. M. Priya, P. Ponnambalam, and K. Muralikumar, "Modular-multilevel converter topologies and applications - a review," IET Pwr. Electr.,vol. 12, no. 2, pp. 170-183, (Feb. 2019).

15. Hagiwara, H. Akagi, and I. Hasegawa, "Start-up and low-speed operation of an electric motor driven by a modular multilevel cascade inverter," IEEE Trans. Ind., Appl., vol. 49, no. 4, pp.15561565, (2013). 